\title{
Key Events in Synaptic Vesicle Endocytosis
}

\author{
Frauke Ackermann, Joshua A. Gregory and Lennart Brodin
}

Additional information is available at the end of the chapter

http://dx.doi.org/10.5772/45785

\section{Introduction}

A synaptic release site is characterized by a pool of synaptic vesicles aggregated to an active zone at the presynaptic plasma membrane. When an action potential arrives, calcium channels in the active zone open to generate a steep increase in calcium concentration. Calcium binds the synaptic vesicle protein synaptotagmin which promotes its interaction with the SNARE complex and with the plasma membrane, together triggering fusion of the synaptic vesicle membrane with the plasma membrane (1). Following fusion, the synaptic vesicle membrane needs to be removed from the plasma membrane to prevent its expansion, and recycling of the vesicle components is needed to refill the pool of vesicles at the release site.

An outline of the steps in the recycling of a synaptic vesicle is depicted in Figure 1. The vesicle membrane first moves out from the active zone into the periactive zone. The mechanism behind such movement is unclear but it is critical in order to maintain the function of the active zone. Impaired clearance of vesicle components from the release site has been linked with depression of neurotransmtter release (2). After the vesicle membrane has reached the periactive zone, clathrin and accessory endocytic factors accumulate to begin the nucleation of a clathrin coat. The coat grows and invaginates until a deeply invaginated coated pit with a narrow neck has formed. The neck of the coat is then surrounded by a dynamin-containing ring or short spiral, which helps to cut off the neck. The free vesicle rapidly sheds its coat and it may be directly refilled with neurotransmitter and prepared for a new round of release. Alternatively, the primary endocytic vesicle may first fuse with an endosome, prior to undergoing a second endosomal budding step to yield a new synaptic vesicle. Although the presence of an endosomal recycling route has been well established $(3,4)$ its precise role in vesicle cycling is not fully clear. It may potentially be used to recycle readily releaseable vesicles (5), or it may participate in refilling the reserve pool during extended periods of synaptic activity (6). The endosomal route may be used more extensively in subsets of synapses (7). 


\section{Clathrin-mediated endocytosis as the main synaptic vesicle recycling pathway}

The model of synaptic vesicle recycling shown in Figure 1 has its origin in quick-freeze studies performed at the frog neuromuscular junction 8 , and microinjection studies performed in giant synapses in lamprey and squid (9-11). In the latter type of experiments a compound - antibody, toxin or peptide - that disrupts the function of an endocytic protein (or a protein-protein interaction) is microinjected into the presynaptic cytoplasm. When the microinjected synapse is examined at rest, the synaptic structure is normal. However, repetitive stimulation uncovers defects in synaptic vesicle recycling. These include loss of synaptic vesicles, expansion of the plasma membrane, and accumulation of clathrin-coated endocytic intermediates in the periactive zone. Depending on which protein is perturbed, the structure of the accumulated intermediates may differ. For instance, if the clathrin/AP2binding region of epsin is perturbed, enlarged coated pits with wide necks occur (Fig. 2). In contrast, if dynamin - SH3 domain interactions are perturbed, deeply invaginated coated pits with narrow necks appear (Fig. 3). If synaptojanin is perturbed, free clathrin coated vesicles accumulate as a sign of impaired uncoating (12).

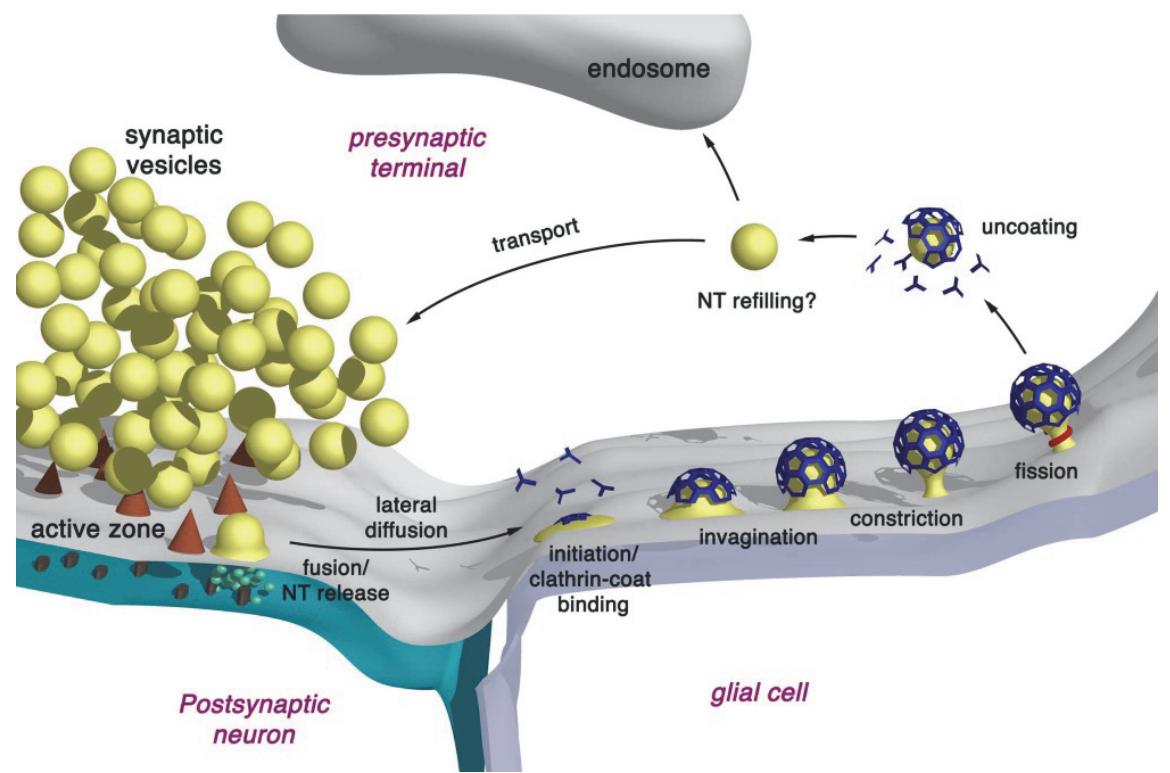

Figure 1. Model of clathrin-mediated synaptic vesicle endocytosis. Synaptic vesicles partially or completely fuse with the presynaptic membrane at the active zone and release neurotransmitter into the synaptic cleft. The membrane of the fused vesicles then diffuses laterally to the areas outside the active zone where it is retrieved by clathrin-mediated endocytosis. Clathrin-coated vesicle formation involves several morphologically distinct steps, from clathrin coat binding, invagination of the coated bud, constriction and fission of the pit 'neck' and the subsequent stripping of the clathrin coat from the newly formed vesicle. The vesicle is then either directly transported back to the cluster of synaptic vesicles or translocated to a primary endosomal compartment. During endocytosis and migration to the release site vesicles are refilled with transmitter (NT). Reproduced from ref 17. 

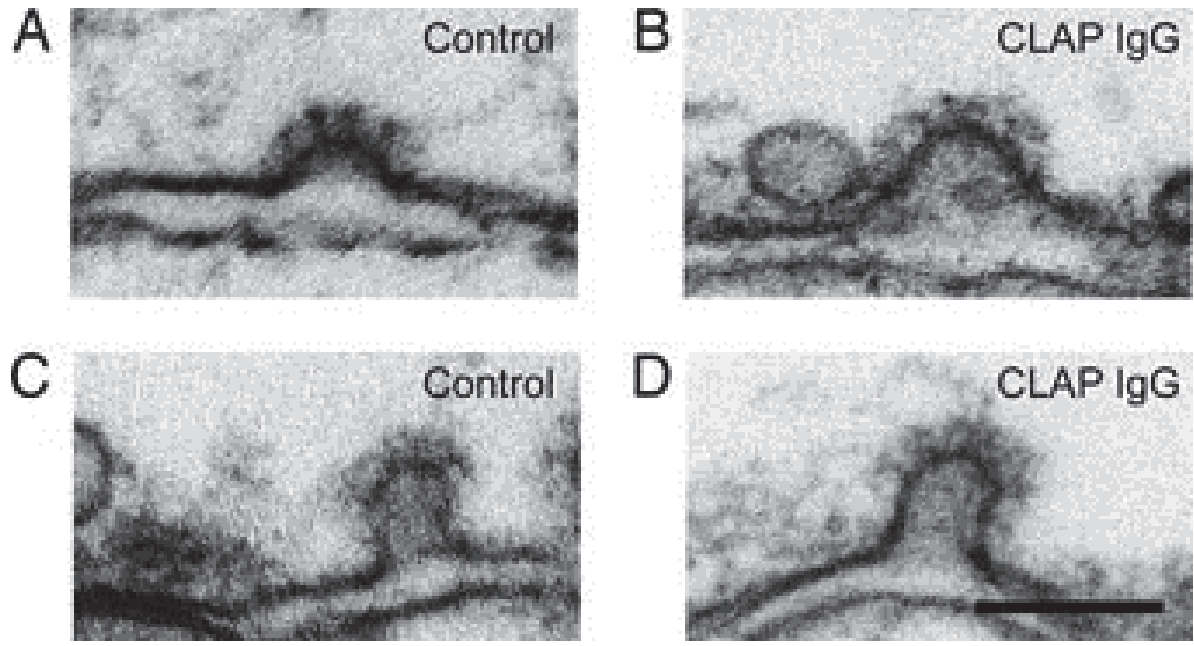

Figure 2. Microinjection of antibodies to the CLAP region of epsin increases the size of coated pits. Electronmicrographs show the periactive zone area in lamprey giant reticulospinal axons stimulated at $5 \mathrm{~Hz}$ following microinjection. A-B, Shallow coated pits from control (A) and CLAP antibody-injected (B). C-D, Examples of non-constricted (bucket-shaped) coated pits from control (C) and CLAP antibodyinjected axons (D). Scale bars $=100 \mathrm{~nm}$. Reproduced from ref 85 .

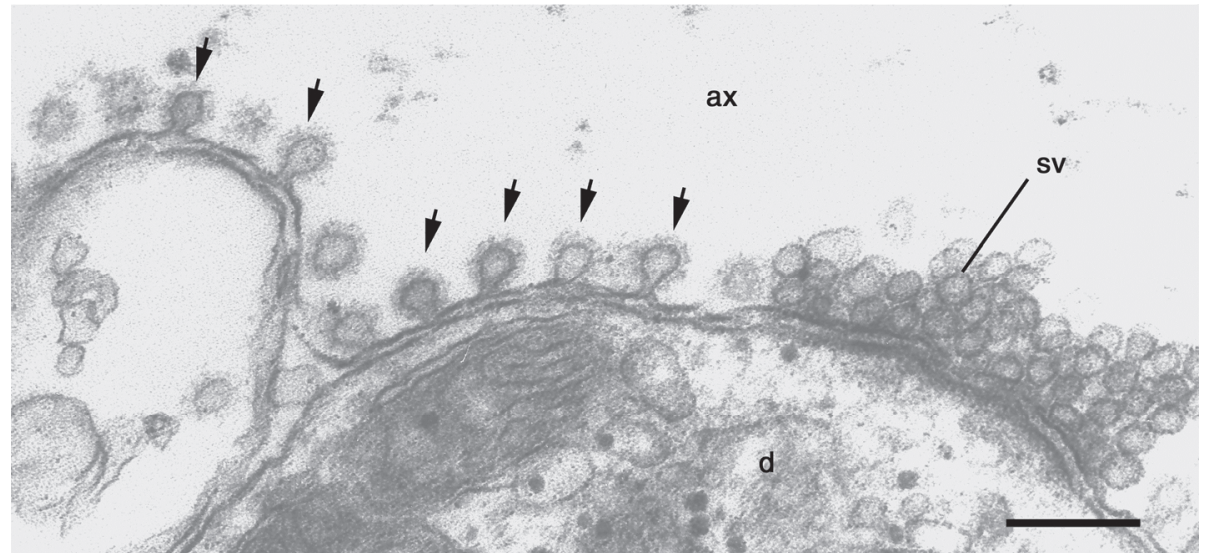

Figure 3. Microinjection of the SH3 domain of amphiphysin traps coated pits with narrow necks. The electronmicrograph shows the periactive zone area in a lamprey giant reticulospinal axon, and a synaptic release site with clustered synaptic vesicles is visible to the right. The axon was stimulated at $0.2 \mathrm{~Hz}$ for $30 \mathrm{~min}$ prior to fixation Scale bar $=200 \mathrm{~nm}$. Reproduced from ref 28 .

The requirement of clathrin in synaptic vesicle recycling has also been demonstrated in experiments using photoinactivation of a transgenically encoded protein (FlAsH-FALI method) $(13,14)$. The technique is based on the use of a short tetracysteine epitope tag that covalently binds a membrane permeable dye, Lumio. When excited with fluorescent light, Lumio inactivates the tagged protein. Following tagging of the clathrin light or heavy chain, 
illumination followed by repetitive stimulation causes a complete loss of synaptic vesicles along with massive accumulation of plasma membrane folds in the terminals $(13,14)$. The importance of clathrin-mediated synaptic vesicle endocytosis has also been demonstrated in real-time imaging experiments using synaptic vesicle proteins tagged with a $\mathrm{pH}$-sensitive reporter (15). Granseth et al showed that brief action potential stimulation is followed by an endocytic response (due to loss of the acidic $\mathrm{pH}$ in the vesicles) with a time course of about $15 \mathrm{~s}$. Such responses were abolished in neurons in which the expression of the clathrin heavy chain had been knocked down by RNAi (16). In addition to the studies mentioned above, a number of genetic studies performed in C. elegans, Drosophila and mice support the critical role of clathrin-mediated synaptic vesicle endocytosis $(15,17)$. In fact, the molecular analysis once began with studies in a temperature-sensitive paralytic Drosophila mutant, shibire $(18,19)$. Following the discovery that the shibire mutation is situated in the dynamin gene $(20,21)$ a network of interconnected endocytic proteins could be identified $(15,22)$.

In the present chapter we will only briefly comment on other, non-clathrin mediated mechanisms of synaptic vesicle recycling. One such mechanism of clathrin-independent membrane internalization is termed bulk endocytosis. Large membrane cisternae are internalized and subsequently converted to synaptic vesicles, but the budding mechanism involved is not well defined. In some model systems, like cerebellar granule cell synapses, bulk endocytosis has been examined in detail and it has been been found to operate under conditions of physiological stimulation (23). In many studies, however, the occurrence of bulk endocytosis in nerve terminals has been linked to non-physiological conditions, including excessive stimulation, or moderate stimulation combined with disruption of the clathrin machinery $(15,24)$. The term kiss-and-run refers to a mode of recycling that involves a transient opening and closing of a fusion pore without loss of the vesicle's integrity. The functional role of kiss-and-run recycling has been the matter of lively debate $(25,26)$. Evidence in favor of the kiss-and-run phenomenon has mainly been obtained in imaging studies. Studies detailing the behavior of single $\mathrm{pH}$-sensitive quantum dots trapped in individual synaptic vesicles in hippocampal boutons supports the possibility that synaptic vesicles can open transiently (27). Further studies, however, are required to determine the generality of this phenomenon and its possible implications for synaptic transmission.

\section{A storage pool of endocytic proteins is associated with synaptic release sites}

Early models of synaptic recycling often assumed that endocytic proteins occur in a diffusible cytoplasmic pool from which they are recruited to the plasma membrane to participate endocytosis. This appears, at least for most proteins, not to be case. In contrast, endocytic proteins have been found to be distinctly accumulated at release sites. Following the observation that an $\mathrm{SH} 3$ domain (of amphiphysin) bound tightly to synaptic vesicle clusters (28), it was shown by immunogold labeling that many endocytic proteins including dynamin, amphiphysin, epsin, endophilin and intersectin accumulate within the vesicle 
cluster (29). The cluster-associated protein pool can be mobilized by synaptic activity. Thus, after a period of repetitive stimulation the endocytic proteins partly dissociate from the synaptic vesicles and appear at the plasma membrane in the periactive zone (30-32). Such protein redistibution has also been observed in live imaging experiments. The clathrin heavy chain is found to be concentrated in the center of release sites at rest but rapidly redistibutes to the periphery upon stimulation (16). Interestingly, not only endocytic, but also other soluble presynaptic proteins, such as synapsin, NSF, rab3, and rabphilin reside in the cluster at rest and redistribute peripherally upon stimulation $(33,34)$. In agreement, in vitro studies showed that these proteins bind reversibly to synaptic vesicles. It was suggested by Denker et al that the presence of a cluster of synaptic vesicles (larger than what is needed to support neurotransmitter release) provides a buffer site for proteins near synaptic release sites (34). What regulates the mobilization of proteins from the synaptic vesicle cluster? The work of Denker et al suggest that calcium is one important factor, but other factors may also be required. In a study of synapsin, Orenbuch et al found that not only calcium influx and phosphorylation of synapsin, but also exocytosis is required in order for synapsin to redistribute from synaptic vesicle clusters (Orenbuch et al J Neurochem, in press). It will be interesting to examine whether mobilization of endocytic proteins from the synaptic vesicle cluster also requires a signal associated with exocytosis.

\section{Early events in synaptic vesicle endocytosis}

The model of synaptic vesicle recycling depicted in Figure 1 may suggest that synaptic vesicle membrane is absent from the periactive zone until it has fused in the active zone and moved laterally. It is now becoming increasingly clear, however, that some synaptic vesicle membrane resides in the axonal plasma membrane in between periods of exo- and endocytosis. Thus, in resting hippocampal nerve terminals, extracellularly applied antibodies to the luminal domain of synaptotagmin binds the axonal surface near release sites (35). Studies employing antibodies with $\mathrm{pH}$-sensitive tags have further shown that a plasma membrane-resident pool of synaptotagmin is preferentially endocytosed at the onset of a bout of endocytosis (36). These findings indicate that a subset of "readily retrievable vesicles" occur in the periactive zone and can be endocytosed rapidly upon stimulation. The protein components in this vesicular membrane pool may be sorted and packaged to facilitate rapid endocytosis (37).

With regard to the precise order of recruitment of endocytic proteins to the periactive zone information is as yet limited. This contrasts with the detailed information that has been obtained in non-neuronal cells grown on glass slides in which protein movement near the plasma membrane can be tracked by total internal reflection (TIRF) microcopy (38). These studies indicate that among the first proteins to occur at the plasma membran is the F-BAR protein FCHo1/2, followed by the scaffold proteins eps15 and intersectin. Different adaptor proteins are then recruited while clathrin shows a slow build-up terminating at scission. Dynamin is present at low levels from early stages but exhibits a peak just before scission. A similar behavior is also observed for endophilin and synaptojanin $(38,39)$. 


\section{What triggers synaptic vesicle endocytosis?}

The simplest answer to the question of what triggers synaptic vesicle endocytosis would be the vesicle membrane itself. It is known that (clathrin-mediated) retrieval of synaptic vesicle membrane can be temporally dissociated from action potential-induced calcium influx (40). Thus, calcium influx is not needed to trigger endocytosis. Moreover, compensatory synaptic vesicle endocytosis can occur after non-calcium-dependent triggering of exocytosis by hypertonic sucrose stimulation (41). The synaptic vesicle membrane thus appears to contain components capable of inducing its reinternalization. However, in the absence of calcium influx, the time-course of endocytosis is slower than that seen under normal conditions of calcium-triggered release. Indeed, several studies have shown that calcium can accelerate endocytosis (42,43). Several proteins have been implicated as calcium sensors for endocytosis, including calmodulin (44), calcineurin (45) and synaptotagmin (46). At present, it remains unclear whether different synapses utilize different trigger mechanisms. One of the most detailed investigations of an endocytic calcium sensor was recently performed in hippocampal neurons (41). These authors examined synaptotagmin, the trigger of fast synchronous exocytosis $(47,48)$, which also is also implicated in endocytosis (49). Interestingly, Yao et al found that the calcium dependence of synaptotagmin in exo- and endocytosis could be uncoupled. Either the $\mathrm{C} 2 \mathrm{~A}$ or $\mathrm{C} 2 \mathrm{~B}$ domain of synaptotagmin could function as calcium sensor for endocytosis, whereas only the C2B domain effectively supported exocytosis. It was also found that retargeting of synaptotagmin to the plasma membrane abolished the calcium dependence of endocytosis but not that of exocytosis. Synaptotagmin thus appears two play distinct roles, one as a calcium sensor that triggers fast synchronous exocytosis and another as a calcium sensor that speeds up endocytosis.

\section{Recycling of SNARE proteins}

The role of SNARE (soluble NSF attachment protein receptors) proteins in synaptic vesicle fusion have been described in great detail (50), but the subsequent fate of the SNARE complex and its components synaptobrevin, syntaxin and SNAP25 have been less well studied. Initial studies suggested that disassembly of the SNARE proteins occurs shortly before fusion such that NSF is in a position to regulate the kinetics of neurotransmitter release (51). More recent studies, however, suggest that SNARE complex disasembly occurs much earlier, even before synaptic vesicle endocytosis. Imaging studies showed that syntaxin remains in the plasma membrane after synaptic vesicles have been endocytosed, indicating that complex dissasembly preceeds endocytosis (52). Moreover, it was shown that NSF and SNARE proteins accumulate in the periactive zone after inhibition of NSF function 53. It is quite possible that, following its disassembly, synaptobrevin participates in clathrinmediated endocytosis. Synaptic vesicle endocytosis is impaired in synaptobrevin-deficient mice (54), and the endocytic adaptors AP180 and CALM have been found to bind synaptobrevin. Notably, these adaptors bind at a site within the SNARE domain that is only accessible after the SNARE complex has been disassembled. Together these observations indicate that SNARE complex disassembly occurs within the plasma membrane of the periactive zone prior to the onset of synaptic vesicle endocytosis, and they further suggest that synaptobrevin may facilitate clathrin-mediated endocytosis. 


\section{BAR domains - Membrane benders or membrane binders?}

Proteins with BAR domains have attracted much interest due to the striking structural features of this domain. Two BAR domains form a dimer with a concave surface that can bind phospholipid membranes $(22,55)$. Hence BAR domains have been implicated as inducers of curvature and as curvature-sensing modules that bind membrane domains with a given curvature. Endophilin is the BAR protein that has attracted most interest in the synaptic vesicle field, both because it is expressed in nerve terminals in organisms ranging from worms and flies to mammals, and because it interacts with dynamin and synaptojanin (56). The effect of perturbing endophilin has been tested in many studies, all of which point to an important role of the protein. Endophilin has been suggested to act at multiple steps in synaptic vesicle endocytosis. A role early in the endocytic reaction was suggested by the finding that shallow coated pits can be trapped by endophilin antibody microinjection in the lamprey giant axon. This phenomenon has also seen in Drosophila after genetic reduction of the endophilin levels (57-59). Endophilin has been detected by immunogold labeling at the rim of shallow coated pits (32). These obervations are possibly compatible with a membrane bending role of endophilin at an early stage of endocytosis, but such a function has not yet been supported by studies in mammalian models (see below). Second, a role for endophilin in recruitment of dynamin to the neck of coated pits has been proposed. Endophilin occurs at the proximal part of the neck of coated pits, and peptides competing the endophilin dynamin interaction inhibit formation of dynamin rings as well as subsequent membrane fission (12,32 see also 60). Finally, endophilin has been linked with vesicle uncoating by its interaction with synaptojanin. In the lamprey giant axon perturbation of the endophilin synaptojanin interaction results in accumulation of numerous free clathrin coated vesicles, in addition to deeply invaginated coated pits. In mice lacking all three endophilin genes nerve terminals were found to contain large numbers of free clathrin coated vesicles (that are nearly absent in wild-type animals) (61). Somewhat surprisingly, in the mouse model no other type of endocytic intermediate was accumulated. Moreover, in both C. elegans and Drosophila the phenotype of synaptojanin mutants closely resembled that of endophilin mutants, and endophilin was found to be required for localization of synaptojanin to nerve terminals $(62,63)$. It is therefore likely that a principal function of endophilin in nerve terminals is to mediate recruitment of synaptojanin to the vesicle neck to support uncoating. Hence, a role of the BAR domain of endophilin as a binder rather then a bender appears most plausible.

\section{New insights into dynamin function and membrane fission}

Different models have been proposed to account for the role of dynamin in catalyzing endocytic membrane fission $(64,65)$. The most recent models incorporate rich highresolution structural information. The crystal structure of full-length dynamin has been determined by taking advantage of assembly-deficient mutants $(66,67)$. Insight into the organization of assembled dynamin multimers has been gained by computer docking of domain crystal structures into cryo-EM images (68). These studies suggest that initial constriction of the coated pit neck, triggered by GTP binding and structural changes in the middle domain of dynamin, promotes GTP domain dimerization between tetramers in adjacent helical rungs. Assembly-stimulated GTP hydrolysis is suggested to induce a 
rotation that provides force, and propagation of this change could cause further constriction of the neck leading to fission. Moreover, in vitro studies have provided detailed insight into the dynamic behavior of dynamin at membranes. It was found that the extended dynamin spirals that form around lipid tubules in the absence of GTP (69) do not effectively promote fission. Instead assembly of short spirals followed by disassembly led to membrane fission (70). Moreover, dynamin alone can form self-limited assemblies that drive vesiculation from a lipid surface in the presence of GTP 71 (Fig. 5A).

Vesiculation

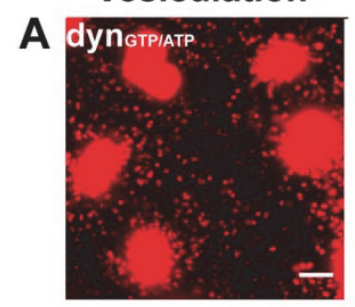

B dyn+EHD GPPATP
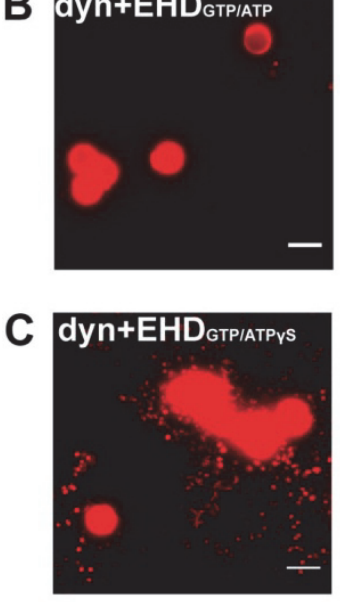
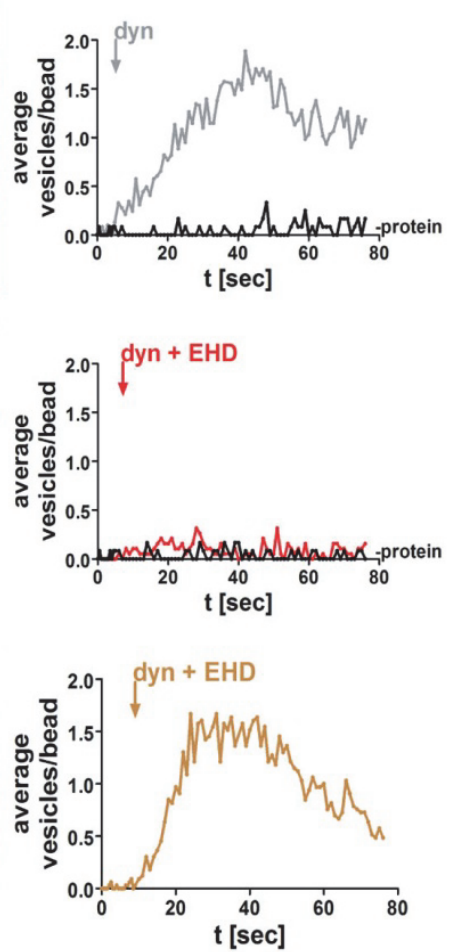

Tubulation
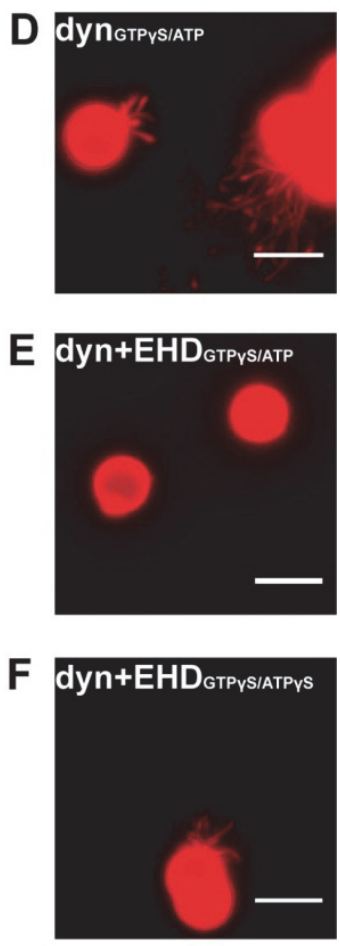

Figure 4. Dynamin-induced vesiculation and its modulation by EHD. A, Vesiculation in vitro from rhodamine-labeled SUPER templates induced by application of dynamin in the constant presence of GTP (1 mM GTP and ATP present in A and B). The trace with the response to dynamin (dyn) is superimposed on the trace preceeding addition of dynamin (-protein) B, Vesiculation was suppressed when dynamin was co-applied with 1-EHD. C, Reduced inhibitory effect of 1-EHD on dynamin-induced vesiculation in the constant presence of GTP after replacement of ATP with ATP $\gamma \mathrm{S}(1 \mathrm{mM})$. D, Application of dynamin in the constant presence of GTP $\gamma \mathrm{S}$ induced formation of narrow tubules $(1 \mathrm{mM}$ GTP $\gamma$ S and ATP present in D-F). E, Tubule formation was suppressed when dynamin was co-applied with l-EHD. F, Reduced inhibitory effect of l-EHD on dynamin-induced tubulation in the constant presence of GTP $\gamma \mathrm{S}$ after replacement of ATP with ATP $\gamma$ S. Scale bars $=5 \mu \mathrm{m}$. Reproduced from ref 75 .

Under in vivo conditions the function of dynamin depends strictly on interactions with other proteins. In particular, interactions with $\mathrm{SH} 3$ domains are important. As indicated above, 
perturbation of dynamin - SH3 domain interactions blocks endocytosis at a late stage (Fig. 3). In this case, dynamin rings do not form suggesting that $\mathrm{SH} 3$ interactions are needed for proper recruitment or assembly of dynamin at the neck of the coated pit. Several proteins may mediate $\mathrm{SH} 3$ domain interactions with dynamin in nerve terminals, including amphiphysin, endophilin, intersectin, SNX9 and syndapin. It may be noted, however, that in Drosophila (and possibly in other invertebrates) neither amphiphysin nor syndapin are expressed in nerve terminals $(72,73)$.

Recent studies performed in the lamprey giant reticulospinal synapse indicate that extrinsic proteins not only regulate the recruitment of dynamin, but they may also control the length of the dynamin spiral. Eps15 homology domain-containing proteins (EHDs) are conserved ATPases implicated in membrane remodelling, primarily in endosomal traffic. EHD1 is enriched at synaptic release sites (74), suggesting a possible involvement in the trafficking of synaptic vesicles. The role of EHD in this function has been analyzed in the lamprey giant reticulospinal synapse. EHD1/3 was detected by immunogold at endocytic structures adjacent to release sites. In antibody microinjection experiments, perturbation of EHD inhibited synaptic vesicle endocytosis and caused accumulation of clathrin-coated pits with atypical, elongated necks (Fig. 5). The necks were covered with helix-like material containing dynamin (75). To test whether EHD directly interferes with dynamin function, fluid supported bilayers were used as in vitro assay. EHD strongly inhibited vesicle budding induced by dynamin in the constant presence of GTP (Fig. 4A-C). EHD also inhibited dynamin-induced membrane tubulation in the presence of GTP $\gamma \mathrm{S}$ (Fig. 4D-E) a phenomenon linked with dynamin helix assembly. Taken together the in vivo and in vitro results suggest that l-EHD acts to limit the formation of long, unproductive dynamin helices, thereby promoting vesicle budding (75).
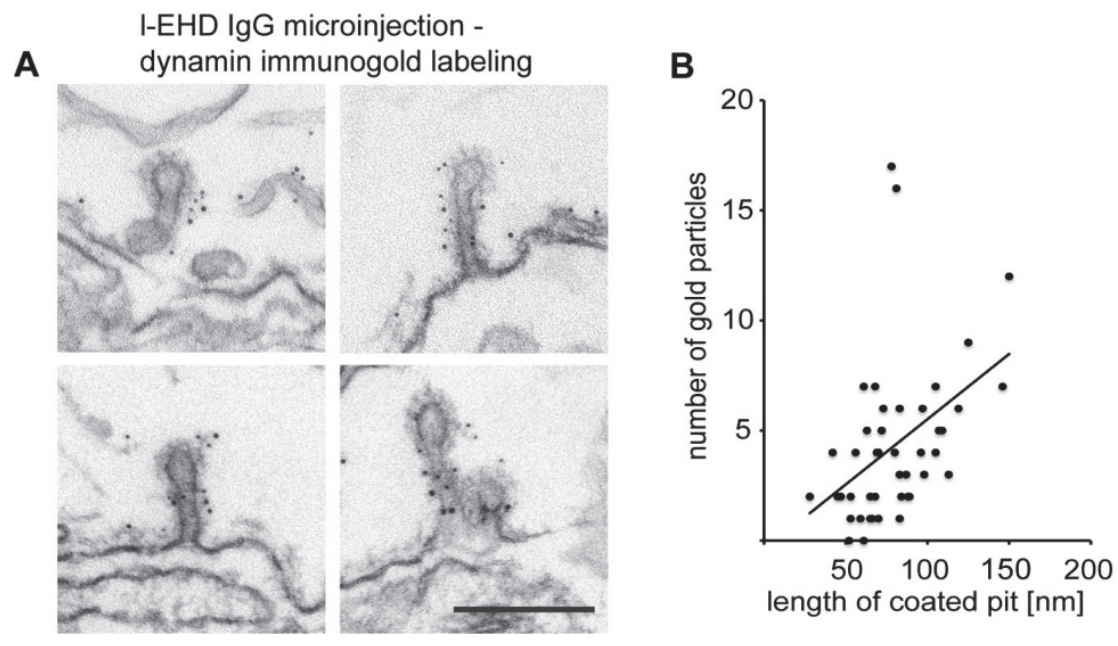

Figure 5. Immunogold localization of dynamin at endocytic pits with elongated necks trapped after perturbation of EHD. A, Examples of coated pits with long necks decorated with dynamin immunogold labeling in lamprey giant axons stimulated after microinjection of EHD antibodies. Scale bar $=0.2 \mu \mathrm{m}$. B, Regression analysis of dynamin labeling and length of coated pits in EHD antibody-injected axons $\left(R^{2}=0.43, n=45,0.05<p<0.01\right.$, Pearson's correlation coefficient). Reproduced from ref 75 . 


\section{Possible implications of synaptic vesicle endocytosis for disease mechanisms}

Knowledge about the mechanisms of synaptic vesicle endocytosis is not only important for our understanding of synaptic information processing, but may potentially also shed light on pathogenetic mechanisms. One aspect conerns toxins and infectious agents that may hijack the synaptic endocytic machinery to enter neurons (76-78). Another aspect concerns the possible role of endocytosis in neurodegenerative disorders that affect synapses. For instance, Lewy body pathology, occurring in Parkinson and other disorders, may involve endocytic uptake of $\alpha$-synuclein fibrils that induce intracellular fibril formation, which in turn leads to synaptic dysfunction (79). Synaptic endocytosis has also been implicated in the pathogenesis of Alzheimer's disease. For instance, part of the processing of the amyloid precursor protein (APP), into synaptotoxic amyloid beta peptides appears to occur in nerve terminals in an endocytosis-dependent manner. Thus, microdialysis studies have shown that the extracellular $A \beta$ pool in brain is elevated by enhanced synaptic activity and lowered after inhibition of synaptic endocytosis (80-82), Accordingly, formation of $A \beta$ in a neuronal cell line has been shown to be suppressed by knock-down of the clathrin adaptor AP180 (83). It is also interesting to note that $\gamma$-secretase, that cleaves APP, is present in nerve terminals (84).

\section{Author details}

Frauke Ackermann, Joshua A. Gregory and Lennart Brodin

Department of Neuroscience, Karolinska Institutet, Stockholm, Sweden

\section{References}

[1] Wojcik, S. M. \& Brose, N. Regulation of membrane fusion in synaptic excitation-secretion coupling: speed and accuracy matter. Neuron 55, 11-24 (2007).

[2] Neher, E. What is Rate-Limiting during Sustained Synaptic Activity: Vesicle Supply or the Availability of Release Sites. Front Synaptic Neurosci 2, 144, doi:10.3389/fnsyn.2010.00144 (2010).

[3] Wucherpfennig, T., Wilsch-Brauninger, M. \& Gonzalez-Gaitan, M. Role of Drosophila Rab5 during endosomal trafficking at the synapse and evoked neurotransmitter release. J Cell Biol 161, 609-624 (2003).

[4] Uytterhoeven, V., Kuenen, S., Kasprowicz, J., Miskiewicz, K. \& Verstreken, P. Loss of skywalker reveals synaptic endosomes as sorting stations for synaptic vesicle proteins. Cell 145, 117-132 (2011).

[5] Hoopmann, P. et al. Endosomal sorting of readily releasable synaptic vesicles. Proc Natl Acad Sci U S A 107, 19055-19060 (2010).

[6] Voglmaier, S. M. \& Edwards, R. H. Do different endocytic pathways make different synaptic vesicles? Curr Opin Neurobiol 17, 374-380 (2007).

[7] Nakatsu, F. et al. Defective function of GABA-containing synaptic vesicles in mice lacking the AP-3B clathrin adaptor. J Cell Biol 167, 293-302 (2004). 
[8] Miller, T. M. \& Heuser, J. E. Endocytosis of synaptic vesicle membrane at the frog neuromuscular junction. Journal of Cell Biology 98, 685-698 (1984).

[9] Brodin, L., Löw, P. \& Shupliakov, O. Sequential steps in clathrin-mediated synaptic vesicle endocytosis. Curr Opin Neurobiol 10, 312-320. (2000).

[10] Brodin, L. \& Shupliakov, O. Giant reticulospinal synapse in lamprey: molecular links between active and periactive zones. Cell Tissue Res 326, 301-310 (2006).

[11] Augustine, G. J. et al. Clathrin and synaptic vesicle endocytosis: studies at the squid giant synapse. Biochem Soc Trans 34, 68-72 (2006).

[12] Gad, H. et al. Fission and uncoating of synaptic clathrin-coated vesicles are perturbed by disruption of interactions with the SH3 domain of endophilin. Neuron 27, 301-312 (2000).

[13] Heerssen, H., Fetter, R. D. \& Davis, G. W. Clathrin dependence of synaptic-vesicle formation at the Drosophila neuromuscular junction. Curr Biol 18, 401-409 (2008).

[14] Kasprowicz, J. et al. Inactivation of clathrin heavy chain inhibits synaptic recycling but allows bulk membrane uptake. J Cell Biol 182, 1007-1016 (2008).

[15] Dittman, J. \& Ryan, T. A. Molecular circuitry of endocytosis at nerve terminals. Annu Rev Cell Dev Biol 25, 133-160 (2009).

[16] Granseth, B., Odermatt, B., Royle, S. J. \& Lagnado, L. Clathrin-mediated endocytosis is the dominant mechanism of vesicle retrieval at hippocampal synapses. Neuron 51, 773786 (2006).

[17] Shupliakov, O. \& Brodin, L. Recent insights into the building and cycling of synaptic vesicles. Exp Cell Res 316, 1344-1350 (2010).

[18] Kelly, L. E. \& Suzuki, D. T. The effects of increased temperature on electroretinograms of temperature-sensitive paralysis mutants of Drosophila melanogaster. Proc Natl Acad Sci U S A 71, 4906-4909 (1974).

[19] Poodry, C. A. \& Edgar, L. Reversible alteration in the neuromuscular junctions of Drosophila melanogaster bearing a temperature-sensitive mutation, shibire. J Cell Biol 81, 520-527 (1979).

[20] Chen, M. S. et al. Multiple forms of dynamin are encoded by shibire, a Drosophila gene involved in endocytosis. Nature 351, 583-586 (1991).

[21] van der Bliek, A. M. \& Meyerowitz, E. M. Dynamin-like protein encoded by the Drosophila shibire gene associated with vesicular traffic. Nature 351, 411-414 (1991).

[22] Frost, A., Unger, V. M. \& De Camilli, P. The BAR domain superfamily: membranemolding macromolecules. Cell 137, 191-196 (2009).

[23] Cousin, M. A. Activity-dependent bulk synaptic vesicle endocytosis--a fast, high capacity membrane retrieval mechanism. Mol Neurobiol 39, 185-189 (2009).

[24] Gad, H., Löw, P., Zotova, E., Brodin, L. \& Shupliakov, O. Dissociation between Ca2+triggered synaptic vesicle exocytosis and clathrin-mediated endocytosis at a central synapse. Neuron 21, 607-616 (1998).

[25] Granseth, B., Odermatt, B., Royle, S. J. \& Lagnado, L. Comment on "The dynamic control of kiss-and-run and vesicular reuse probed with single nanoparticles". Science 325, 1499; author reply 1499 (2009).

[26] Zhang, Q., Li, Y. \& Tsien, R. W. The dynamic control of kiss-and-run and vesicular reuse probed with single nanoparticles. Science 323, 1448-1453 (2009). 
[27] Park, H., Li, Y. \& Tsien, R. W. Influence of Synaptic Vesicle Position on Release Probability and Exocytotic Fusion Mode. Science, (2012, in press).

[28] Shupliakov, O. et al. Synaptic vesicle endocytosis impaired by disruption of dynaminSH3 domain interactions. Science 276, 259-263 (1997).

[29] Shupliakov, O. The synaptic vesicle cluster: a source of endocytic proteins during neurotransmitter release. Neuroscience 158, 204-210 (2009).

[30] Evergren, E. et al. Amphiphysin is a component of clathrin coats formed during synaptic vesicle recycling at the lamprey giant synapse. Traffic 5, 514-528 (2004).

[31] Evergren, E. et al. Intersectin is a negative regulator of dynamin recruitment to the synaptic endocytic zone in the central synapse. J Neurosci 27, 379-390 (2007).

[32] Sundborger, A. et al. An endophilin-dynamin complex promotes budding of clathrincoated vesicles during synaptic vesicle recycling. J Cell Sci 124, 133-143 (2011).

[33] Chi, P., Greengard, P. \& Ryan, T. A. Synapsin dispersion and reclustering during synaptic activity. Nat Neurosci 4, 1187-1193 (2001).

[34] Denker, A., Kröhnert, K., Bückers, J., Neher, E. \& Rizzoli, S. O. The reserve pool of synaptic vesicles acts as a buffer for proteins involved in synaptic vesicle recycling. Proc Natl Acad Sci U S A (in press) (2011).

[35] Fernandez-Alfonso, T., Kwan, R. \& Ryan, T. A. Synaptic vesicles interchange their membrane proteins with a large surface reservoir during recycling. Neuron 51, 179-186 (2006).

[36] Hua, Y. et al. A readily retrievable pool of synaptic vesicles. Nat Neurosci 14, 833-839, (2011).

[37] Willig, K. I., Rizzoli, S. O., Westphal, V., Jahn, R. \& Hell, S. W. STED microscopy reveals that synaptotagmin remains clustered after synaptic vesicle exocytosis. Nature 440, 935939 (2006).

[38] Taylor, M. J., Perrais, D. \& Merrifield, C. J. A high precision survey of the molecular dynamics of mammalian clathrin-mediated endocytosis. PLoS Biol 9, e1000604, doi:10.1371/journal.pbio.1000604 (2011).

[39] Henne, W. M. et al. FCHo proteins are nucleators of clathrin-mediated endocytosis. Science 328, 1281-1284 (2010).

[40] Gad, H., Low, P., Zotova, E., Brodin, L. \& Shupliakov, O. Dissociation between Ca2+triggered synaptic vesicle exocytosis and clathrin-mediated endocytosis at a central synapse. Neuron 21, 607-616 (1998).

[41] Yao, J., Kwon, S. E., Gaffaney, J. D., Dunning, F. M. \& Chapman, E. R. Uncoupling the roles of synaptotagmin I during endo- and exocytosis of synaptic vesicles. Nat Neurosci 15, 243-249 (2012).

[42] Hosoi, N., Holt, M. \& Sakaba, T. Calcium dependence of exo- and endocytotic coupling at a glutamatergic synapse. Neuron 63, 216-229 (2009).

[43] Balaji, J., Armbruster, M. \& Ryan, T. A. Calcium control of endocytic capacity at a CNS synapse. J Neurosci 28, 6742-6749 (2008).

[44] $\mathrm{Wu}, \mathrm{X}$. S. et al. $\mathrm{Ca}(2+)$ and calmodulin initiate all forms of endocytosis during depolarization at a nerve terminal. Nat Neurosci 12, 1003-1010 (2009).

[45] Clayton, E. L. et al. The phospho-dependent dynamin-syndapin interaction triggers activity-dependent bulk endocytosis of synaptic vesicles. J Neurosci 29, 7706-7717 (2009). 
[46] Nicholson-Tomishima, K. \& Ryan, T. A. Kinetic efficiency of endocytosis at mammalian CNS synapses requires synaptotagmin I. Proc Natl Acad Sci U S A 101, 1664816652,(2004).

[47] Littleton, J. T., Stern, M., Schulze, K., Perin, M. \& Bellen, H. J. Mutational analysis of Drosophila synaptotagmin demonstrates its essential role in $\mathrm{Ca}(2+)$-activated neurotransmitter release. Cell 74, 1125-1134 (1993).

[48] Geppert, M. et al. Synaptotagmin I: a major Ca2+ sensor for transmitter release at a central synapse. Cell 79, 717-727 (1994).

[49] Zhang, J. Z., Davletov, B. A., Sudhof, T. C. \& Anderson, R. G. Synaptotagmin I is a high affinity receptor for clathrin AP-2: implications for membrane recycling. Cell 78, 751-760 (1994).

[50] Sudhof, T. C. \& Rothman, J. E. Membrane fusion: grappling with SNARE and SM proteins. Science 323, 474-477 (2009).

[51] Schweizer, F. E. et al. Regulation of neurotransmitter release kinetics by NSF. Science 279, 1203-1206 (1998).

[52] Mitchell, S. J. \& Ryan, T. A. Syntaxin-1A is excluded from recycling synaptic vesicles at nerve terminals. J Neurosci 24, 4884-4888 (2004).

[53] Yu, W., Kawasaki, F. \& Ordway, R. W. Activity-dependent interactions of NSF and SNAP at living synapses. Mol Cell Neurosci 47, 19-27 (2011).

[54] Deak, F., Schoch, S., Liu, X., Sudhof, T. C. \& Kavalali, E. T. Synaptobrevin is essential for fast synaptic-vesicle endocytosis. Nat Cell Biol 6, 1102-1108 (2004).

[55] McMahon, H. T. \& Gallop, J. L. Membrane curvature and mechanisms of dynamic cell membrane remodelling. Nature 438, 590-596 (2005).

[56] Ringstad, N., Nemoto, Y. \& De Camilli, P. The SH3p4/Sh3p8/SH3p13 protein family: binding partners for synaptojanin and dynamin via a Grb2-like Src homology 3 domain. Proceedings of the National Academy of Sciences of the United States of America 94, 8569-8574 (1997).

[57] Ringstad, N. et al. Endophilin/SH3p4 is required for the transition from early to late stages in clathrin-mediated synaptic vesicle endocytosis. Neuron 24, 143-154 (1999).

[58] Guichet, A. et al. Essential role of endophilin A in synaptic vesicle budding at the Drosophila neuromuscular junction. EMBO J 21, 1661-1672, doi:10.1093/emboj/21.7.1661 (2002).

[59] Andersson, F., Low, P. \& Brodin, L. Selective perturbation of the BAR domain of endophilin impairs synaptic vesicle endocytosis. Synapse 64, 556-560 (2010).

[60] Fabian-Fine, R. et al. Endophilin promotes a late step in endocytosis at glial invaginations in Drosophila photoreceptor terminals. J Neurosci 23, 10732-10744 (2003).

[61] Milosevic, I. et al. Recruitment of endophilin to clathrin-coated pit necks is required for efficient vesicle uncoating after fission. Neuron 72, 587-601 (2011).

[62] Verstreken, P. et al. Synaptojanin is recruited by endophilin to promote synaptic vesicle uncoating. Neuron 40, 733-748 (2003).

[63] Schuske, K. R. et al. Endophilin is required for synaptic vesicle endocytosis by localizing synaptojanin. Neuron 40, 749-762 (2003).

[64] Hinshaw, J. E. Dynamin and its role in membrane fission. Annu Rev Cell Dev Biol 16, 483-519. (2000). 
[65] Marks, B. et al. GTPase activity of dynamin and resulting conformation change are essential for endocytosis. Nature 410, 231-235. (2001).

[66] Faelber, K. et al. Crystal structure of nucleotide-free dynamin. Nature 477, 556-560 (2011).

[67] Ford, M. G., Jenni, S. \& Nunnari, J. The crystal structure of dynamin. Nature 477, 561-566 (2011).

[68] Chappie, J. S. et al. A pseudoatomic model of the dynamin polymer identifies a hydrolysis-dependent powerstroke. Cell 147, 209-222 (2011).

[69] Danino, D. \& Hinshaw, J. E. Dynamin family of mechanoenzymes. Curr Opin Cell Biol 13, 454-460. (2001).

[70] Bashkirov, P. V. et al. GTPase cycle of dynamin is coupled to membrane squeeze and release, leading to spontaneous fission. Cell 135, 1276-1286 (2008).

[71] Pucadyil, T. J. \& Schmid, S. L. Real-time visualization of dynamin-catalyzed membrane fission and vesicle release. Cell 135, 1263-1275 (2008).

[72] Zhang, B. \& Zelhof, A. C. Amphiphysins: raising the BAR for synaptic vesicle recycling and membrane dynamics. Bin-Amphiphysin-Rvsp. Traffic 3, 452-460 (2002).

[73] Kumar, V., Alla, S. R., Krishnan, K. S. \& Ramaswami, M. Syndapin is dispensable for synaptic vesicle endocytosis at the Drosophila larval neuromuscular junction. Mol Cell Neurosci 40, 234-241 (2009).

[74] Wei, S. H. et al. EHD1 is a synaptic protein that modulates exocytosis through binding to snapin. Molecular and Cellular Neuroscience 45, 418-429 (2010).

[75] Jakobsson, J. et al. Regulation of synaptic vesicle budding and dynamin function by an EHD ATPase. J Neurosci 31, 13972-1398 (2011).

[76] Binz, T. \& Rummel, A. Cell entry strategy of clostridial neurotoxins. J Neurochem 109, 1584-1595 (2009).

[77] Montal, M. Botulinum neurotoxin: a marvel of protein design. Annu Rev Biochem 79, 591-617, doi:10.1146/annurev.biochem.051908.125345 (2010).

[78] Harper, C. B. et al. Dynamin inhibition blocks botulinum neurotoxin type A endocytosis in neurons and delays botulism. J Biol Chem 286, 35966-35976 (2011).

[79] Volpicelli-Daley, L. A. et al. Exogenous alpha-synuclein fibrils induce Lewy body pathology leading to synaptic dysfunction and neuron death. Neuron 72, 57-71 (2011).

[80] Kamenetz, F. et al. APP processing and synaptic function. Neuron 37, 925-937, doi:S0896627303001247 [pii] (2003).

[81] Cirrito, J. R. et al. Synaptic activity regulates interstitial fluid amyloid-beta levels in vivo. Neuron 48, 913-922, (2005).

[82] Cirrito, J. R. et al. Endocytosis is required for synaptic activity-dependent release of amyloid-beta in vivo. Neuron 58, 42-51, (2008).

[83] Wu, F., Matsuoka, Y., Mattson, M. P. \& Yao, P. J. The clathrin assembly protein AP180 regulates the generation of amyloid-beta peptide. Biochem Biophys Res Commun (2009).

[84] Frykman, S. et al. Synaptic and endosomal localization of active gamma-secretase in rat brain. PLoS One 5, e8948 (2010).

[85] Jakobsson, J. et al. Role of epsin 1 in synaptic vesicle endocytosis. Proc Natl Acad Sci U S A 105, 6445-6450 (2008). 